Author's Note: This is the final accepted version of the manuscript for online distribution. The following article is published as:

Palmisano, S., Apthorp, D., Seno, T., \& Stapley, P.J. (2014). Spontaneous postural sway predicts the strength of smooth vection. Experimental Brain Research, 232, 1185-1191. doi: 10.1007/s00221-014-3835-y

If you have subscription rights to the journal, the published version is available at: http://link.springer.com/article/10.1007\%2Fs00221-014-3835-y

\title{
SPONTANEOUS POSTURAL SWAY PREDICTS THE STRENGTH OF SMOOTH VECTION
}

\author{
Stephen Palmisano ${ }^{1}$, Deborah Apthorp ${ }^{2}$, Takeharu Seno ${ }^{3} \&$ Paul J. Stapley ${ }^{4}$ \\ ${ }^{1}$ School of Psychology, University of Wollongong, Australia \\ ${ }^{2}$ Research School of Psychology, Australian National University, Australia \\ ${ }^{3}$ Faculty of Design, Kyushu University, Japan \\ ${ }^{4}$ School of Medicine, University of Wollongong, Australia
}

\section{Corresponding author:}

Stephen Palmisano, School of Psychology, University of Wollongong, NSW, Australia, 2522

Tel: (612) 4221-3640

Fax: (612) 4221-4163

Email: stephenp@uow.edu.au

Short title: Postural Sway and Vection

Keywords: self-motion, vection, postural sway, vision, optic flow 
This study asked whether individual differences in the influence of vision on postural stability could be used to predict the strength of subsequently induced visual illusions of self-motion (vection). In the experiment, we first measured spontaneous postural sway while subjects stood erect for $60 \mathrm{~s}$ with their eyes both open and both closed. We then showed our subjects two types of self-motion display: radially expanding optic flow (simulating constant velocity forwards self-motion) and vertically oscillating radially expanding optic flow (simulating constant velocity forwards self-motion combined with vertical head oscillation). As expected, subjects swayed more with their eyes closed (compared to open) and experienced more compelling illusions of self-motion with vertically oscillating (as opposed to smooth) radial flow. The extent to which participants relied on vision for postural stability - measured as the ratio of sway with eyes closed compared to open - was found to predict vection strength. However, this was only the case for displays representing smooth self-motion. It seems that for oscillating displays, other factors, such as visualvestibular interactions, may be more important. 


\section{INTRODUCTION}

Many of the sensory systems involved in perceiving our self-motions (vision, the vestibular system of the inner ear, the proprioceptive detection of limb/joint movement and position, and the somatosensory system of cutaneous receptors) also provide essential information for controlling our stance (Howard 1982; Palmisano et al. 2011). Vision, in particular, is known to play important roles in both types of processing. This can be seen clearly by the fact that visual motion of large regions of the surrounding environment can induce both illusory self-motion (known as vection) and significant postural responses in standing observers (van Asten et al. 1988; Berthoz et al. 1975, 1979; Lee and Aronson 1974; Lee and Lishman 1975; Lestienne et al. 1977; Lishman and Lee 1973; Stoffregen 1985). For example, Lishman and Lee (1973) found that compelling back-and-forth vection could be quickly induced by moving the walls and ceiling of an experimental 'swinging room' back and forth around their standing observers. Even though the floor on which they stood never moved, these observers swayed noticeably in a manner consistent with their visually perceived self-motion. Subsequent research showed that the magnitudes of vection and visually induced postural sway both increase with the area, velocity and spatial frequency of the surrounding scene motion (Berthoz et al. 1979; Lestienne et al. 1977). Several researchers have proposed that the two phenomena might even be controlled by the same visual processes (Previc and Mullen 1990; Tanahashi et al. 2007). While such visually induced postural responses may seem to be due to, or compensating for, vection, this is unlikely to be the case since: (1) vection can occur without postural change, (2) visually induced postural sway can occur without vection, and (3) vection is typically experienced several seconds after the onset of visually induced postural sway (e.g. Berthoz et al. 1979; Previc and Mullen 1990). This does not, however, rule out the possibility of a common underlying mechanism which might be at work prior to the sensation of 'self-motion' reaching consciousness. Thus, the goal of visually induced sway could still be to minimise any global optical flow so as to maintain an upright posture (Palmisano et al. 2009). 
A number of recent studies have examined possible links between visually induced postural responses and vection. In one study, Kuno and colleagues (1999) measured the magnitudes of the sway and vection induced when standing subjects were exposed to large frontal displays simulating alternating forwards and backwards self-motion in depth. They reported that increases in visually induced postural sway were typically followed shortly afterwards by increases in linear vection. Similarly, another study by Thurrell and Bronstein (2002), which exposed standing subjects to rotary optic flow, also observed greater magnitude postural sway during roll vection than during perceived object/scene motion. Most recently, Tanahashi and colleagues (2007) found that while postural sway was greatest during roll vection, visual scene rotation without vection still generated more postural sway than no visual motion conditions. In order to explain their results, they proposed that self-motion perception and postural control both utilised the same visual information, but that their respective mechanisms had different operating thresholds.

Over the last decade, evidence has been mounting that postural measures can be used identify people who will be susceptible to visually induced motion sickness (e.g. Smart et al. 2002; Stoffregen and Smart 1998). Inspired by these reports, the current experiment examined whether individual differences in the influence of vision on postural stability might be used to predict the strength of subsequently experienced vection. Unlike most of the studies above, which measured vection and visually induced postural responses continuously throughout the simulated self-motion ${ }^{1}$, here we measured spontaneous postural sway prior to any visually simulated self-motion. In an attempt to identify the contribution of vision to stabilization, we measured the lengths of postural sway paths when subjects were quietly standing on the floor of a brightly lit stationary room, with both eyes open and with both eyes closed (During these postural measurements there was no display motion). Visual stabilization was then estimated by calculating each subject's Romberg quotient (i.e. sway path length with eyes closed divided by sway path length with eyes open - see Van Parys and Njiokiktjien 1976; Kelly et al. 2008).

\footnotetext{
${ }^{1}$ Simultaneous measurement of vection and sway runs the risk that act of making the vection rating (whether it be verbal, button or joystick based) might introduce artefactual postural responses. In any event, there is a phase lag between the onset of visually induced postural sway and the subsequently reported onset of vection (see Kuno et al., 1999).
} 
The purpose of this study was to determine whether individual differences in this Romberg quotient could predict the strength of the vection induced by two different types of optic flow: (1) smooth radial flow, which simulated $6 \mathrm{~m} / \mathrm{s}$ forward self-motion in depth; and (2) vertically oscillating radial flow, which simulated the same constant velocity forward self-motion combined with vertical head oscillation (frequency $1.5 \mathrm{~Hz}$; amplitude $0.75 \mathrm{~m}$ ). While smooth optic flow is thought to generate only minimal/transient conflicts between the stationary observer's visual and non-visual self-motion sensory systems, vertically oscillating radial flow should generate substantial intersensory conflict (since the vertical self-accelerations simulated would normally be accompanied by significant and continuing vestibular stimulation). Despite these expectations, it is well established that adding horizontal/vertical simulated viewpoint oscillation to smooth radial flow significantly increases vection in depth (Kim et al. 2012; Palmisano et al. 2008; 2011; 2012). In fact, adding simulated viewpoint oscillation to smooth flow is now a proven method for enhancing the vection experience (when all other aspects of the display are held constant - e.g. smooth flow speed, display density, etc. - see also Nakamura, 2010, 2013). Thus, the dual benefit of the current "smooth versus oscillating" radial flow manipulation was that we could not only examine the relationship between postural sway and vection for different strength visual inducing stimuli, but we could also examine it under different conditions of (expected) intersensory conflict as well.

In the current context, we hypothesized that vection might be stronger for individuals who rely more on their vision for postural stability, and that they would also display larger oscillation advantages for vection the idea being that these individuals might be both more prone to experiencing visually-mediated illusory self-motion and less sensitive to any potential non-visual sensory conflicts arising when they viewed the oscillating self-motion displays.

\section{METHODS}


Subjects. Twenty psychology students and staff (16 females and 4 males; mean age $25.9, \mathrm{SD}=9.5$ ) from the University of Wollongong participated in this experiment. The 18 undergraduate psychology students received course credit for their participation. All had normal or corrected-to-normal vision and no existing vestibular or neurological impairments. The Wollongong University Ethics Committee approved the study in advance (HE12/249). Each subject provided written informed consent before participating in the experiment. These protocols were in accordance with the ethical standards laid down in the 1964 Declaration of Helsinki.

Apparatus. Computer-generated displays were rear projected onto a flat projection screen (1.48 m wide $\mathrm{x}$ $1.20 \mathrm{~m}$ high) using a Mitsubishi Electric (Model XD400U) data colour projector (1024 (horizontal) $\times 768$ (vertical) pixel resolution). When viewed from a distance of $0.65 \mathrm{~m}$ away from the screen, these displays subtended a visual angle of $66 \mathrm{deg} \times 62 \mathrm{deg}$. During this experiment, subjects stood on a Bertec balance plate (http://bertec.com/products/balance-plates.html) which recorded changes to the location of their center of foot pressure (CoP sampled at $1000 \mathrm{~Hz})$.

Visual Motion Stimuli. Our radially expanding optic flow displays all simulated forwards self-motion at 6 $\mathrm{m} / \mathrm{s}$. The simulated self-motion passed through a 3-D cloud consisting of 2000 randomly placed blue circular objects (each being 8 pixels in diameter). These objects were uniformly distributed throughout the simulated 3-D environment - the dimensions of which were $15 \mathrm{~m}$ wide $\times 15 \mathrm{~m}$ high $\times 40 \mathrm{~m}$ deep ${ }^{2}$. In half of the displays, simulated vertical viewpoint oscillation was also added to the radial flow display. When present, this simulated viewpoint oscillation had a frequency of $1.5 \mathrm{~Hz}$ and an amplitude of $0.75 \mathrm{~m}$.

Procedure. Subjects initially provided potentially relevant demographic details, such as their age, any health issues, alcohol and medication consumption, etc. They next removed their shoes and had their height, weight, hip width, foot length and foot width recorded. We then asked subjects to step onto the force

\footnotetext{
${ }^{2}$ It should be noted that while these simulations were consistent with a $6 \mathrm{~m} / \mathrm{s}$ forward self-motion through an environment that was $15 \mathrm{~m}$ wide $\times 15 \mathrm{~m}$ high $\times 40 \mathrm{~m}$ deep, they were also consistent with an unlimited number of other velocity/size combinations.
} 
plate and marked their foot positions (feet together) with a whiteboard marker to ensure that they did not change during testing. During CoP measurement, subjects were asked to stand still with their knees kept straight and their hands folded on their abdomen. We measured changes to their centre of foot pressure (CoP, sampled at $1000 \mathrm{~Hz}$ ) while they stood erect on a Bertec balance plate for periods of $60 \mathrm{~s}$ (twice with their eyes open looking at the part of the stationary room that was directly in front of them, and twice with their eyes closed). Only after the CoP measurements were obtained for these quiet stance conditions, did we expose the standing subjects to three purely radial and three vertically oscillating radial flow displays. The order in which these six vection displays were presented was counterbalanced across the twenty subjects (either the three purely radial displays, or the three oscillating radial displays, were presented first). At the end of each $30 \mathrm{~s}$ vection display, subjects verbally rated the strength of their vection experience on a 100 point scale (with ' 0 ' indicating no perceived self-motion). They were then exposed to a blank display for 1 minute aimed at extinguishing any residual postural aftereffects, as well as allowing subjects time to recover prior to their next vection trial.

\section{RESULTS}

Postural Sway Data

The CoP data (in $\mathrm{m}$ ) were first smoothed using a low-pass order-5 Butterworth filter with a cut-off frequency of $10 \mathrm{~Hz}$ (to remove unwanted high-frequency artefacts - see Figure 1 for an example). This smoothed CoP data was then converted into sway path length estimates for each eyes-open or eyes-closed trial. Sway path length was calculated as the total distance travelled (in $\mathrm{m}$ ) by the CoP over a $60 \mathrm{~s}$ period, using the following formula:

$$
P L=\sum_{n-1}^{N} \sqrt{[x(n)-x(n-1)]^{2}+[y(n)-y(n-1)]^{2}}
$$


where $x$ and $y$ are the horizontal and depth CoP positions, and $n$ is the number of data points (Hufschmidt et al. 1980; Stapley et al. 2006).

\section{$* * * * *$ INSERT FIGURE 1 ABOUT HERE}

As expected from previous research (e.g. Lee and Lishman 1975; Smart et al. 2002), a paired samples t-test revealed that mean sway paths were significantly longer when the subjects eyes were closed $(\mathrm{M}=1.11 \mathrm{~m}$, $S D=0.31 \mathrm{~m})$ compared to when their eyes were open $(M=0.61 \mathrm{~m}, \mathrm{SD}=0.13 \mathrm{~m}), t_{19}=10.40, p<0.0001$. In order to ascertain the contribution of vision to postural stability, we divided each subject's mean eyesclosed sway path by his/her mean eyes-open sway path - generating their Romberg quotient. Our subjects' Romberg quotients ranged from a minimum of 1.35 to a maximum of 2.66 . According to Kelly et al. (2008) a Romberg quotient of more than 1.0 indicates a stabilizing influence of vision.

\section{Vection Strength Data}

As expected, a paired samples t-test revealed that oscillating radial flow $(\mathrm{M}=60.4, \mathrm{SD}=21.4)$ induced significantly stronger vection than smooth radial flow $(\mathrm{M}=49.2, \mathrm{SD}=22.4), t_{19}=2.22, p=0.038$. In order to test our first hypothesis that individual differences in the influence of vision on postural stability might predict vection strength, we then performed two separate linear regression analyses (with the subjects' Romberg quotients as the predictor and either their smooth or oscillating vection strength ratings as the dependent variable). We first confirmed that in both cases the assumptions of normality, linearity and homoscedasticity of the residuals were met. While the relationship between Romberg quotients and vection strength ratings was not significant in the oscillating radial flow conditions, $R^{2}=0.000, t_{19}=0.05, p=$ 0.96 (see Figure 2 right), higher Romberg quotients significantly predicted stronger vection ratings in the smooth radial flow conditions, $R^{2}=0.36, t_{19}=3.15, p=0.006$ (see Figure 2 left). A Spearman's Rank Order correlation was run in order to determine whether the latter significant relationship was due to the 
influence of outliers ${ }^{3}$. Contrary to this notion, we found that the relationship between Romberg quotients and smooth vection remained statistically significant $\left(r_{s}(18)=.554, p=0.011\right)$.

\section{$* * * * *$ INSERT FIGURE 2 ABOUT HERE}

In order to check that the above relationship was not simply due to individual differences in spontaneous postural sway (as opposed to individual differences in the Romberg quotient) we ran two additional linear regression analyses. However, the relationship between eyes-open sway path and vection strength ratings was not significant for either oscillating radial flow $\left(R^{2}=0.056, t_{19}=1.03, p=0.32\right)$ or smooth radial flow $\left(R^{2}\right.$ $\left.=0.004, t_{19}=0.268, p=0.79\right)$.

\section{$* * * * *$ INSERT FIGURE 3 ABOUT HERE $* * * * *$}

Next we tested our second hypothesis that individuals who rely more on their vision for postural stability would also display larger oscillation advantages for vection. By subtracting each subject's vection strength ratings for the smooth radial flow from their vection strength ratings for oscillating radial flow, we obtained an index of the size of their oscillation advantage for vection. As can be seen in Figure 3, the Romberg quotient significantly predicted the degree of this oscillation advantage for vection, $R^{2}=0.34, t_{19}=3.15, p=$ 0.006. However, opposite to the predictions of our second hypothesis, we found that subjects who relied on vision less for stabilization actually tended to experience larger oscillation advantages for vection.

\section{$* * * * *$ INSERT FIGURE 4 ABOUT HERE}

Thus, it was also possible that the failure to find a significant relationship between the Romberg quotients and oscillating vection could have arisen due to ceiling effects. There was some support for this proposal.

\footnotetext{
${ }^{3}$ Spearman's rho is robust to the influence of outliers, as it limits the contribution of each outlier simply to the value of its rank.
} 
As can be seen in Figure 4, subjects who experienced stronger vection with smooth radial flow tended to display smaller oscillation advantages than those who had experienced weaker vection with smooth radial flow $\left(R^{2}=0.3, t 19=-0.275, p=0.013\right.$.

\section{DISCUSSION}

Consistent with our expectations and the findings of previous studies, spontaneous postural sway was significantly greater during eyes-closed (as opposed to open) conditions and oscillating displays induced significantly more compelling vection than smooth displays. Previously, under superficially similar conditions (i.e. standing bare-foot on a force plate with and without vision), Lacour and colleagues (1997) found that almost half of their healthy control subjects displayed decreased postural sway upon eye closure, while the remaining subjects displayed either no change or increased postural sway. By contrast, we found that vision always reduced spontaneous postural sway in our 20 participants to either a greater or lesser extent (with Romberg quotients that were always greater than one, ranging from a minimum of 1.35 to a maximum of 2.66$)$.

Importantly, these observed individual differences in Romberg quotients were here found to significantly predict the strength of the (less compelling) vection induced by smooth radial flow. However, they did not appear to predict the strength of the (more compelling) vection induced by oscillating radial flow. As was noted above, the higher the Romberg quotient, the more vision appeared to contribute to the subject's postural stability. While subjects with higher Romberg quotients were found on average to report stronger vection during smooth radial flow displays, the vection induced by oscillating radial flow appeared to be largely independent of these Romberg quotients.

Romberg quotients were, however, found to significantly predict the size of our subjects' oscillation advantages for vection - with lower Romberg quotients being associated with larger oscillation advantages. Subjects with low Romberg quotients reported weaker vection during smooth radial flow and displayed 
greater vection improvements when simulated viewpoint oscillation was added to their radial inducing displays. By contrast, the oscillation advantage was considerably less for subjects with higher Romberg quotients.

In the introduction, we had hypothesized that subjects with larger Romberg quotients (i.e. vision more important for stabilization) would also display larger oscillation advantages for vection. The idea was that subjects who relied more on vision would be less sensitive to the non-visual sensory conflicts generated when viewing oscillating radial flow displays while standing on a stationary ground surface. Contrary to this hypothesis, we found that subjects with lower Romberg quotients (i.e. vision less important for stabilization) tended to experience larger oscillation advantages for vection. It is not clear then how differential sensitivity to sensory conflict could explain the current failure of Romberg quotients to predict the strength of vection induced by oscillating radial flow. Instead, we believe that the most likely explanation of the current results is that since subjects with higher Romberg quotients already tended to perceive stronger smooth vection, they had less room for vection enhancement when simulated viewpoint oscillation was added to their inducing displays.

As noted above, the subjects in the present study all displayed reductions in postural sway when their eyes were open. Thus it is possible that our (more visual) subjects might have displayed different relationships between Romberg quotients and vection than other (less visual) subjects whose postural responses were either not altered by vision, or in fact increased by vision. While the current data cannot completely rule out these alternative possibilities, we have recently reported that eyes-open/eyes-closed sway area ratios also predict the strength of smooth vection (Apthorp et al. 2013). Importantly, this new study utilized a completely different sample of subjects to those tested here.

In the last decade, research has shown that several postural measures can be used to identify people who are more susceptible to visually induced motion sickness. For example, Stoffregen and his colleagues (e.g. Smart et al. 2002; Stoffregen and Smart, 1998) have shown that when subjects stand inside a swinging 
room, increases in the variability, range and velocity of their visually induced postural sway precede the onset of sickness symptoms. In a similar swinging room study, Smart and colleagues (2002) were able to predict which of their subjects would eventually become sick (with an accuracy of between $80-92 \%$ ), based solely on individual differences in visually induced postural stability along the axis of the simulated selfmotion.

Importantly, recent studies have also shown that pre-exposure postural responses (e.g. spontaneous postural sway) can be used to identify people who are more or less likely to become sick when later exposed to visual motion stimulation (e.g. Koslucher et al. 2013; Stoffregen and Smart, 1998; Stoffregen et al. 2000; Stoffregen et al. 2010; Villard et al. 2008). Our research here adds significantly to this literature, demonstrating for the first time that pre-exposure postural responses can also be used (at least under some circumstances) to identify people who are more/less likely to benefit from the experience of vection in simulation. 


\section{ACKNOWLEDGEMENTS}

This research was supported by an Australian Research Council Discovery grant to SP (DP0772398). 


\section{REFERENCES}

Apthorp, D., Stapley P, Palmisano S (2013) Individual variations in visual control of posture predict vection. Perception 42 (ECVP Abstract Supplement): 172.

Berthoz A, Lacour M, Soechting JF, Vidal PP (1979) The role of vision in the control of posture during linear motion. Prog Brain Res 50:197-209.Diener HC, Dichgans J, Bacher M, Gompf B (1984) Quantification of postural sway in normals and patients with cerebellar diseases. Electroencephalography and clinical Neurophysiology 54:134-142.

Duarte M, Freitas SMSF (2010) Revision of posturography based on force plate for balance evaluation. Rev Bras Fisioter 14:183-92.

Howard IP, (1982). Human Visual Orientation. Wiley, Chichester.

Hufschmidt A, Dichgans J, Mauritz KH, Hufschmidt M (1980) Some methods and parameters of body sway quantification and their neurological application. Archiv für Psychiatrie und Nervenkrankheiten 228:135150.

Kelly JW, Riecke B, Loomis JM, Beall AC (2008). Visual control of posture in real and virtual environments. Perception and Psychophysics 70:158-165. doi: 10.3758/PP.70.1.158

Kim J, Palmisano S (2008) Effects of active and passive viewpoint jitter on vection in depth. Brain Res Bull 77:335-342. doi:10.1016/j.brainresbull.2008.09.011

Kim J, Palmisano S (2010) Visually-mediated eye-movements regulate the capture of optic flow in selfmotion perception, Exp Brain Res 202:355-361. doi: 10.1007/s00221-009-2137-2

Kim J, Palmisano S, Bonato F (2012) Simulated angular head oscillation enhances vection in depth. Perception 41:402-414. doi:10.1068/p6919

Koslucher F C, Haaland E, Stoffregen T A (2013). Body load and the postural precursors of motion sickness. Gait \& Posture in press.

Kuno S, Kawakita T, Kawakami O, Miyake Y, Watanabe S (1999). Postural adjustment response to depth direction moving patterns produced by virtual reality graphics. The Japanese Journal of Physiology 49:417-424. 
Lacour M, Barthelemy J, Borel L, Magnan J, Xerri C, Chays A, Ouaknine M (1997) Sensory strategies in human postural control before and after unilateral vestibular neurotomy. Exp Brain Res 115:300-310.

Lishman JR, Lee DN (1973) The autonomy of visual kinaesthesis, Perception 2:287-294.

Lee DN, Lishman JR (1975) Visual proprioceptive control of stance. Journal of Human Movement Studies 1:87-95.

Lestienne F, Soechting J, Berthoz A (1977) Postural readjustments Induced by linear motion of visual scenes. Exp Brain Res 28:363-384.

Nakamura S (2010) Additional oscillation can facilitate visually induced self-motion perception: The effect of its coherence and amplitude gradient. Perception 39:320-329. doi:10.1068/p6534

Nakamura S (2013) Effects of additional visual oscillation on vection under voluntary eye movement conditions - retinal image motion is critical in vection facilitation. Perception 42:529-536. doi: $10.1068 / p 7486$

Nishiike S, Nakagawa S, Nakagawa A, Uno A, Tonoike M, Takeda N, Kubo T (2002) Magnetic cortical responses evoked by visual linear forward acceleration, Neuroreport 13:1805-1808.

Palmisano S, Allison RS, Pekin F (2008) Accelerating self-motion displays produce more compelling vection in depth. Perception 37:22-33. doi: 10.1068/p5806

Palmisano S, Bonato F, Bubka A, Folder J (2007) Vertical Display Oscillation Increases Vection in Depth and Simulator Sickness. Aviat, Space and Environ Med 78:951-956. doi: 10.3357/ASEM.2079.2007

Palmisano S, Pinniger GJ, Ash A, Steele JR (2009) Effects of simulated viewpoint jitter on visually induced postural sway. Perception 38:442-453. doi:10.1068/p6159

Palmisano S, Allison RS, Kim J, Bonato F (2011) Simulated Viewpoint jitter shakes sensory conflict accounts of self-motion perception. Seeing \& Perceiving 24:173-200. doi: 10.1163/187847511X570817

Palmisano S, Kim J, Freeman TCA (2012) Horizontal fixation point oscillation and simulated viewpoint oscillation both increase vection in depth. Journal of Vision, 12(12:15), 1-14. doi: 10.1167/12.12.15

Previc FH, Mullen TJ (1990) A comparison of the latencies of visually induced postural change and selfmotion perception. J Vestib Res 1:317-323. 
Smart L, Stoffregen TA, Bardy BG (2002) Visually induced motion sickness predicted by postural instability. Hum Factors 44:451-465. doi: 10.1518/0018720024497745

Stapley P, Beretta MV, Toffola ED, Schieppati M (2006) Neck muscle fatigue and postural control in patients with whiplash injury. Clin Neurophysiol 117:610-622. doi: 10.1016/j.clinph.2005.11.007

Stoffregen TA (1985) Flow structure versus retinal location in the optical control of stance. J of Exp Psychol Human 11:554-565.

Stoffregen TA, Bardy B G, Bonnet CT, Pagulayan RJ (2006). Postural stabilization of visually guided eye movements. Ecological Psychology 18: 191-222.

Stoffregen TA, Hettinger LJ, Haas MW, Roe M, Smart LJ, 2000 Postural instability and motion sickness in a fixed-base flight simulator. Human Factors 42: 458-469.

Stoffregen TA, Smart LJ (1998) Postural instability precedes motion sickness. Brain Res Bull 47:437-448.

Tanahashi S, Ujike H, Kozawa R, Ukai K (2007) Effects of visually simulated roll motion on vection and postural stabilization. J NeuroEng Rehabil 4:1-11. doi:10.1186/1743-0003-4-39

Thurrell AEI, Bronstein AM (2002) Vection increases the magnitude and accuracy of visually evoked postural responses. Exp Brain Res 147:558-560. Doi: 10.1007/s00221-002-1296-1

Van Asten WNJC, Gielen, CCAM, van der Gon JJD (1988) Postural adjustments induced by simulated motion of differently structured environments. Exp Brain Res 73:371-383.

Van Parys JAP, Njiokiktjien CJ (1976) Romberg's sign expressed in a quotient. Agressologie 17B:95-100.

Villard, S.J., Flanagan, M.B., Albanese, G.M. \& Stoffregen, T.A. (2008). Postural instability and motion sickness in a virtual moving room. Human Factors, 50: 332-345. 
Figure 1. Filtered anterior/posterior $(A / P)$ and medial/lateral $(M / L)$ CoP displacements for one representative subject over the course of two separate $60 \mathrm{~s}$ measurement periods (one with eyes open and the other with eyes closed).

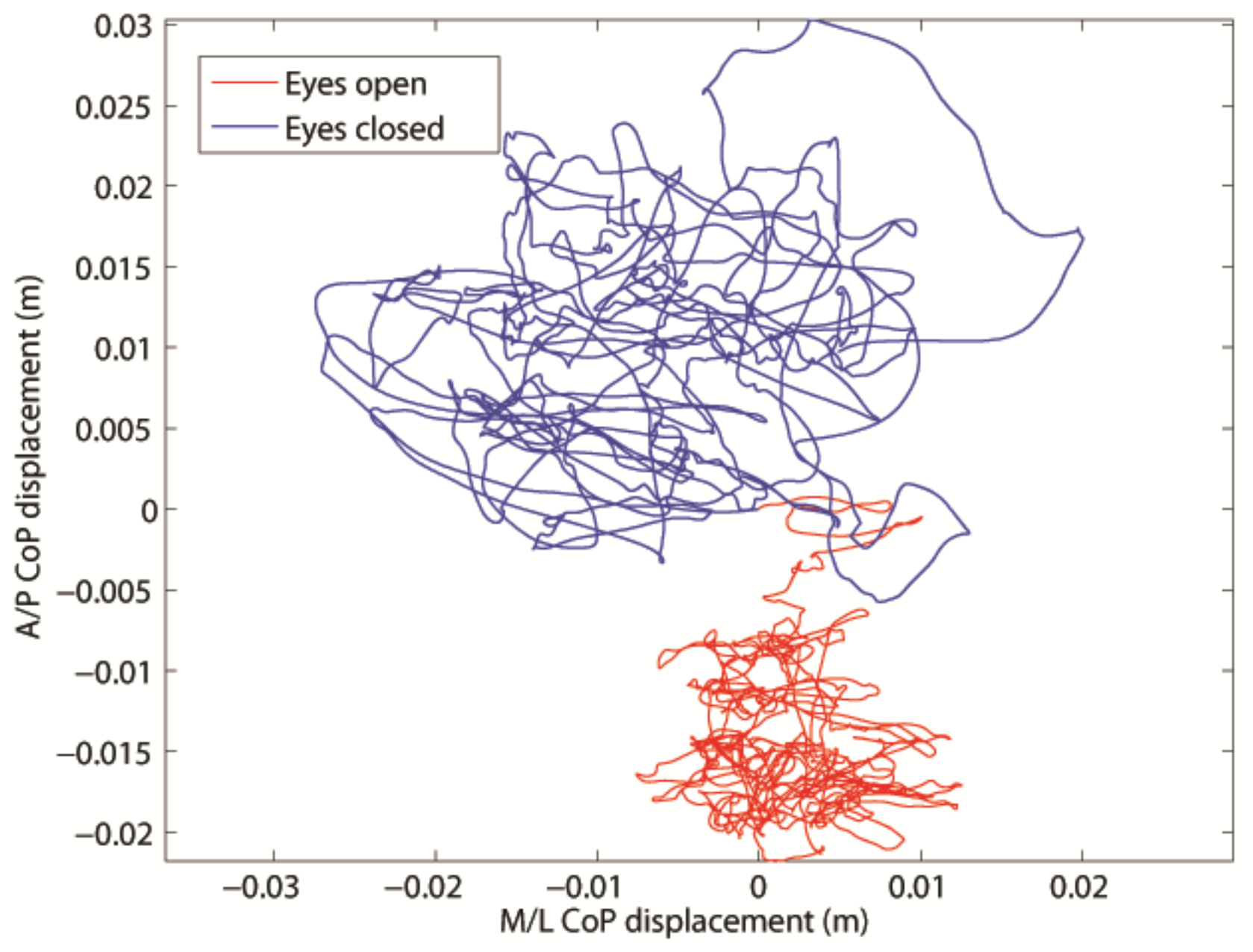


Figure 2. Relationship between the Romberg quotient for sway path and the rated strength of smooth (left) and oscillating (right) vection.

Smooth radial flow

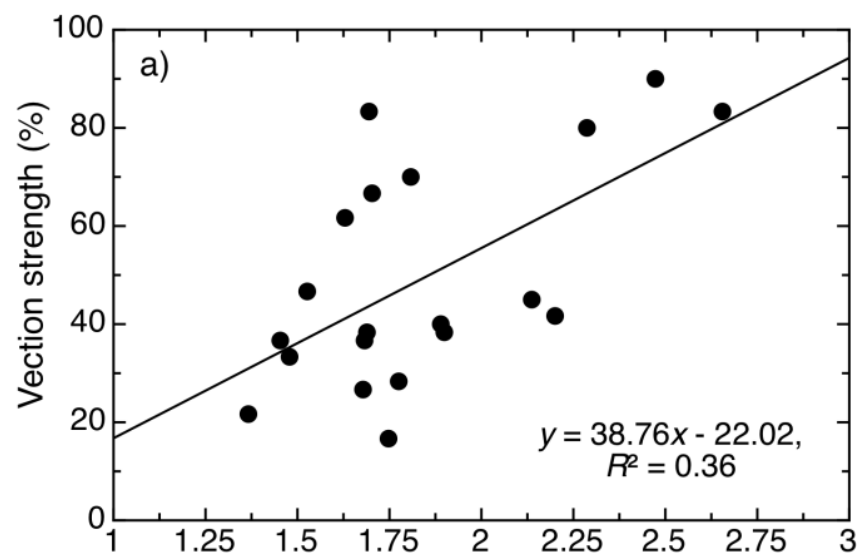

Romberg quotient

\section{Oscillating flow}

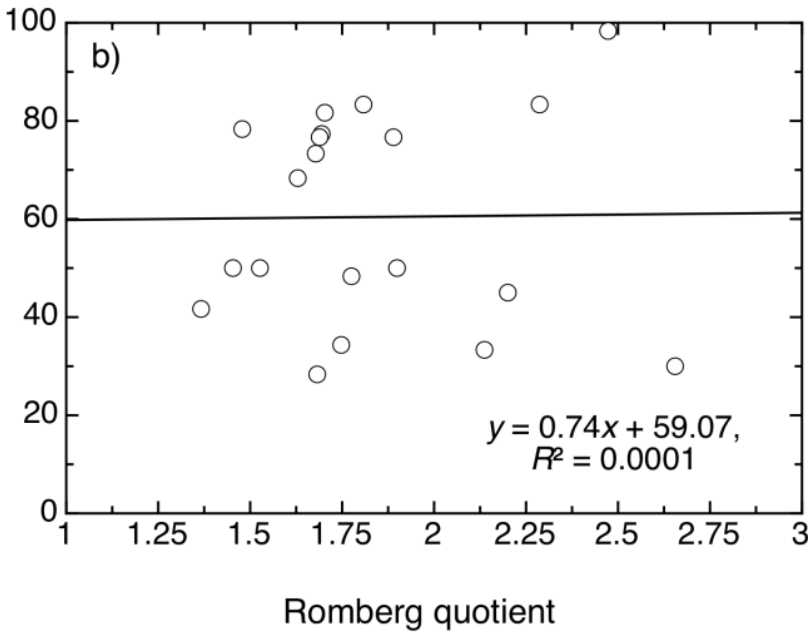


Figure 3. Relationship between the Romberg quotient for sway path and the size of the oscillation advantage for vection (in \%). Four of our 20 subjects did not display an oscillation advantage for vection.

\section{Oscillation advantage}

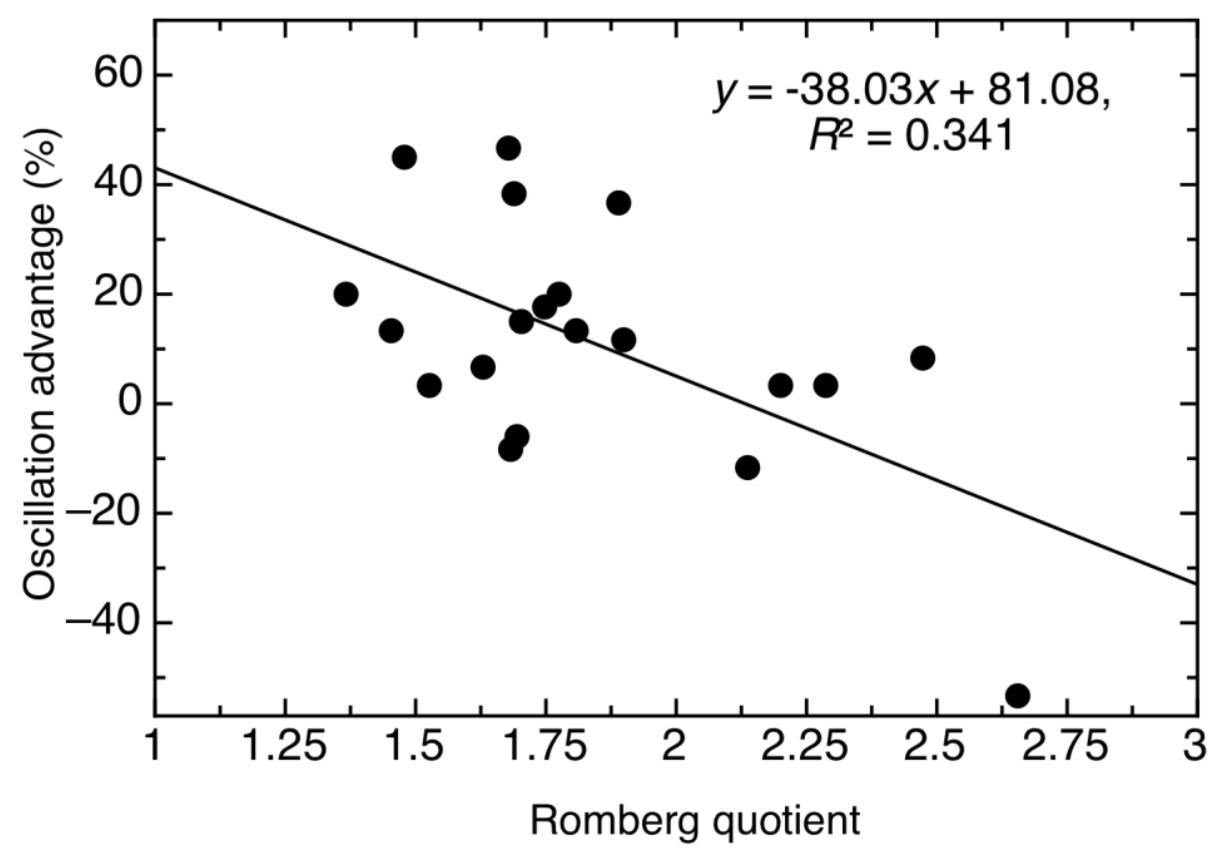


Figure 4. Relationship between smooth vection ratings and the size of the oscillation advantage for vection (in \%).

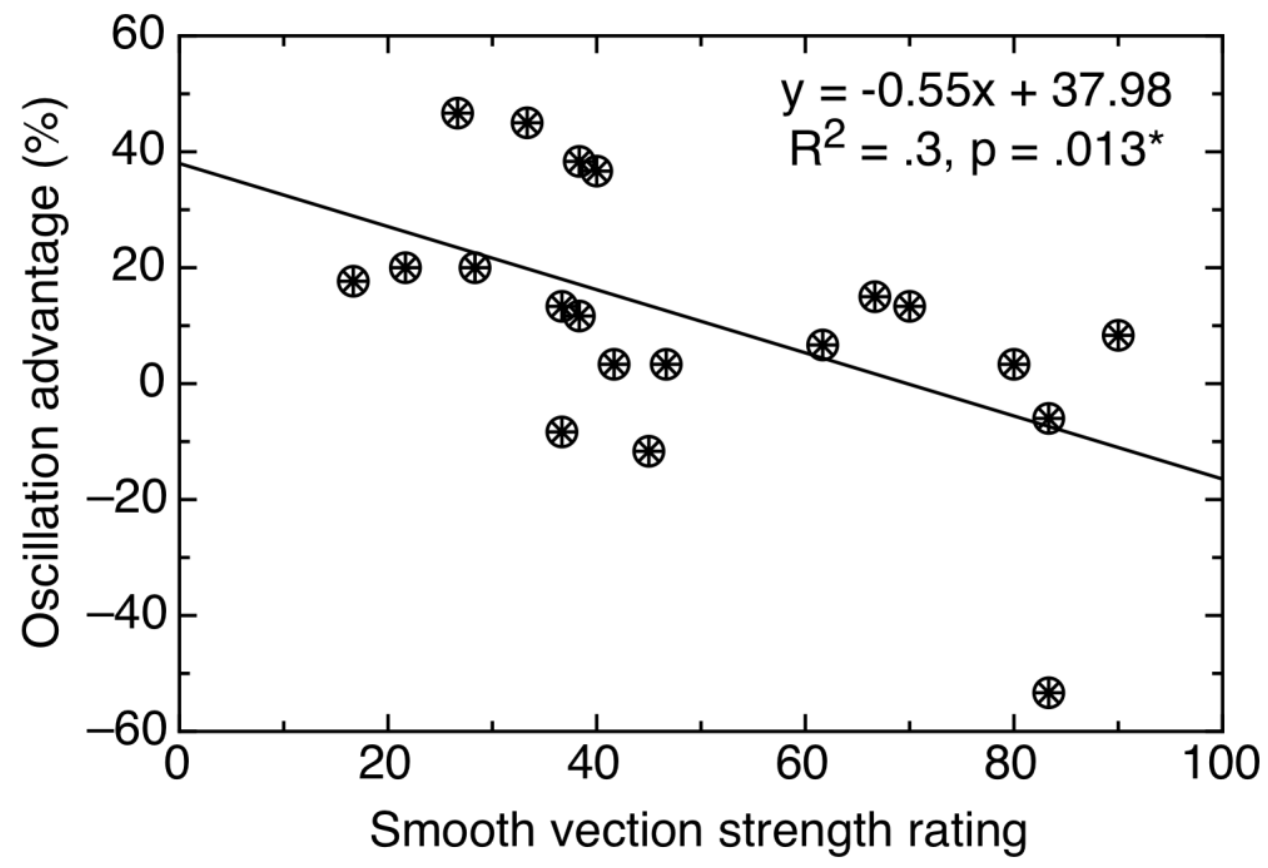

